\title{
Extraction of negative charges from an ion source: transition from an electron repelling to an electron attracting plasma close to the extraction surface
}

Christian Wimmer, ${ }^{1, a)}$ Ursel Fantz, ${ }^{1}$ and the NNBI-team ${ }^{1, b}$ )

Max-Planck-Institut für Plasmaphysik, Boltzmannstr. 2, 85748 Garching,

Germany

(Dated: 5 August 2016)

Large-scale sources for negative hydrogen ions, capable of delivering an extracted ion current of several ten amperes, are a key component of the neutral beam injection system of the upcoming ITER fusion device. Since the created heat load of the inevitably co-extracted electrons after magnetic separation from the extracted beam limits their tolerable amount, special care must be taken for the reduction of coextracted electrons - in particular in deuterium operation, where the larger amount of co-extracted electrons often limits the source performance. By biasing the plasma grid (PG, first grid of the extraction system) positively with respect to the source body the plasma sheath in front of the PG can be changed from an electron repelling towards an electron attracting sheath. In this way, the flux of charged particles onto the PG can be varied, thus changing the bias current and inverse to it the amount of co-extracted electrons. The PG bias affects also the flux of surface-produced $\mathrm{H}^{-}$ towards the plasma volume as well as the plasma symmetry in front of the plasma grid, strongly influenced by an $\vec{E} \times \vec{B}$ drift. The influence of varying PG sheath potential profile on the plasma drift, the negative hydrogen ion density and the source performance at the prototype $\mathrm{H}^{-}$source is presented, comparing hydrogen and deuterium operation. The transition in the PG sheath profile takes place in both isotopes, with a minimum of co-extracted electrons formed in case of the electron attracting PG sheath. The co-extracted electron density in deuterium operation is higher than in hydrogen operation, which is accompanied by an increased plasma density in deuterium.

PACS numbers: 52.27.Cm,52.70.Ds

\footnotetext{
a) christian.wimmer@ipp.mpg.de

b) See authors list of B. Heinemann et al., Fusion Eng. Des. 88, 512 (2013).
} 


\section{INTRODUCTION}

The international ITER fusion experiment requires two neutral beam injectors (NBI)

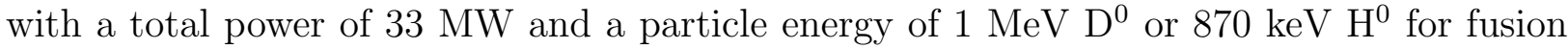
plasma heating and current drive (HNB injectors). ${ }^{1,2}$ Due to the much higher neutralization efficiency in a gas target of the negative ions compared to positive ions the NBI system must be based on a source for negative ions, which are accelerated to the required beam energy and neutralized afterward. Large and powerful sources are required for delivering the required amount of negative hydrogen ion current of $57 \mathrm{~A} \mathrm{D}^{-}$and $66 \mathrm{~A} \mathrm{H}^{-}$, respectively, in order to achieve the required heating power when taking into account losses during the neutralization process as well as during the transport in the extraction system and in the beamline. The European ITER domestic agency F4E has defined an R\&D roadmap in order to fulfill the development of the ITER HNB facility. ${ }^{3}$ A prototype RF source has been developed at the Max-Planck-Institut für Plasmaphysik (IPP) Garching in the past, which has become the ITER reference design in $2007 .{ }^{4}$

The negative hydrogen ion source is based on surface production of negative ions on a caesiated grid: hydrogen atoms and positive ions, produced in a low pressure, low temperature hydrogen plasma are converted to negative hydrogen ions on a surface with low work function. ${ }^{5}$ As a consequence of the vacuum conditions in the source (background pressure in the order of $1 \times 10^{-6}$ mbar) in combination with the high chemical reactivity of Cs, a repetitive or even continuous evaporation of Cs into the source is required for maintaining a low work function of the converter surface. A critical parameter is the amount of co-extracted electrons, which often limits the performance of the prototype source: co-extracted electrons are removed out of the extracted particle beam by magnets, bending them onto the second grid of the three-grid extraction system prior full acceleration. The tolerable heat load on the grid is technologically limited by the cooling system, leading to the requirement for the ratio of extracted particle current densities $j_{\mathrm{e}} / j_{\mathrm{D}^{-}}<1$. Whereas the ITER parameters have been demonstrated for short pulses in hydrogen and deuterium, ${ }^{6}$ the instability of co-extracted electrons in long pulses are a major issue in deuterium operation, where the source can only be run at reduced parameters. ${ }^{7}$ Since $j_{\mathrm{e}}$ is a critical parameter, a better understanding of the electron dynamics close to the extraction system is highly desirable.

The amount of co-extracted electrons is reduced by two mechanisms: firstly, a sepa- 
ration of the plasma production region in a so-called driver by inductive RF coupling $\left(n_{\mathrm{e}} \approx 10^{18} \mathrm{~m}^{-3}, T_{\mathrm{e}} \approx 10 \mathrm{eV}\right)$ from the $\mathrm{H}^{-}$production and extraction region by an expansion chamber with a magnetic filter field applied reduces both, the plasma electron temperature and electron density, by one order of magnitude each. The reduction of the electron temperature is of particular importance in order to minimize the $\mathrm{H}^{-}$destruction process by electron stripping. The magnetic filter field acts as an electron filter, which strongly reduces the diffusion perpendicular to the field lines compared to the diffusion parallel to the field lines. Thus it is obvious that the reduction of $j_{\mathrm{e}}$ depends on the magnetic filter field topology and field strength. However, the applied horizontal filter field ( $\vec{x}$ direction in the coordinate system shown in figure 1 ) also leads to a vertical ( $\vec{y}$ direction) plasma drift in the expansion volume towards the plasma grid. ${ }^{8}$ Secondly, a positive bias of the caesiated plasma grid (PG, first grid of the extraction system facing towards the plasma chamber) with respect to the source body decreases efficiently the amount of co-extracted electrons. The positive effect of biasing the plasma grid in negative ion sources is known since the 1980 s and is used in many different ion sources ${ }^{9-13}$; the general effect on the source performance has been reported also for the BATMAN (Bavarian Test Machine for Negative Ions) test facility in earlier publications ${ }^{6,14}$. Variation of the bias voltage changes the potential profile in the sheath in front of the plasma grid from an electron repelling case at low bias towards an electron attracting sheath at high bias. Thus, charged particle fluxes from the bulk plasma towards the PG as well as the flux of surface produced negative hydrogen ions from the PG towards the bulk plasma are influenced. The PG behaves as a large probe, strongly influencing the plasma in front of it. The I-V characteristic results in large currents up to several $10 \mathrm{~A}$. The co-extracted electron current reacts inverse to the bias current $\left(j_{\mathrm{e}}\right.$ is high at low bias current $I_{\text {bias }}$ and vice versa). Because the plasma potential in front of the plasma grid is influenced due to the large bias current, the axial electric field distribution in the source is modified. This results in a change of the $\vec{E} \times \vec{B}$ drift and consequently in a variation of the vertical ( $\vec{y}$ direction) plasma distribution in front of the plasma grid. It should be noted that although the $\vec{E} \times \vec{B}$ drift plays an important role, the total plasma drift is more complex and consists of several components - theoretical models indicate that in addition the diamagnetic drift plays an important role for the plasma transport through the magnetic filter. ${ }^{8,15,16}$ Although neutral Cs is distributed homogeneously in the source during plasma phases ${ }^{17}$, the plasma drift might affect the vertical distribution of $\mathrm{Cs}^{+}$ions 
due to a higher rate of ionization collisions in drift direction. A so called bias plate (same potential as the source body) covers the area of the PG where no extraction apertures are positioned and thus increases the area on source potential. ${ }^{6}$

The focus of this paper is to correlate the influence of the bias on the plasma and source performance as an effect of the varying sheath potential profile: the effect of the transition from an electron repelling towards an electron attracting PG sheath on the source performance, the vertical plasma symmetry and the positive and negative ion density is presented for measurements carried out at the prototype source of the BATMAN test facility ${ }^{6}$ in hydrogen and deuterium operation.

In negative hydrogen ion sources, a virtual cathode can be present in the PG sheath, ${ }^{18,19}$ also affecting the electron dynamics from the plasma onto the PG surface. Since the plasma potential at BATMAN can be determined in the bulk plasma only, the PG sheath potential difference is used for the description of the sheath property, neglecting the presence of a virtual cathode. Thus, the results on varying PG sheath profile have to be seen as general, relative trends and not as a direct influence of absolute numbers in the PG sheath potential difference.

\section{EXPERIMENTAL SETUP}

The BATMAN test facility is equipped with the 1/8 ITER size prototype ion source. An important aim of BATMAN is the investigation of processes of the ion source close to the PG due to its flexible diagnostic access. BATMAN is a pulsed test facility allowing for plasma phases of typically $6 \mathrm{~s}$ including a beam extraction phase of $4 \mathrm{~s}$. The vacuum phase between two pulses has a duration of about 3-4 minutes. During this experimental campaign, BATMAN has not been operated with the internal filter magnets (standard configuration), but using a more flexible magnetic setup with the permanent magnets mounted in an external frame $^{20}$. The magnets have been positioned at an axial distance of $9 \mathrm{~cm}$ to the PG. The polarity of the field can be changed resulting in a vertically ( $\vec{y}$ direction) symmetric change of the plasma drift direction to either the top part ('drift up') or to the bottom part ('drift down') close to the PG. All measurements presented in this paper have been carried out in the 'drift up' setting of the filter field, which is pointing from left to right in beam direction.

A sketch of the ion source including the measurement positions is shown in figure 1 . 

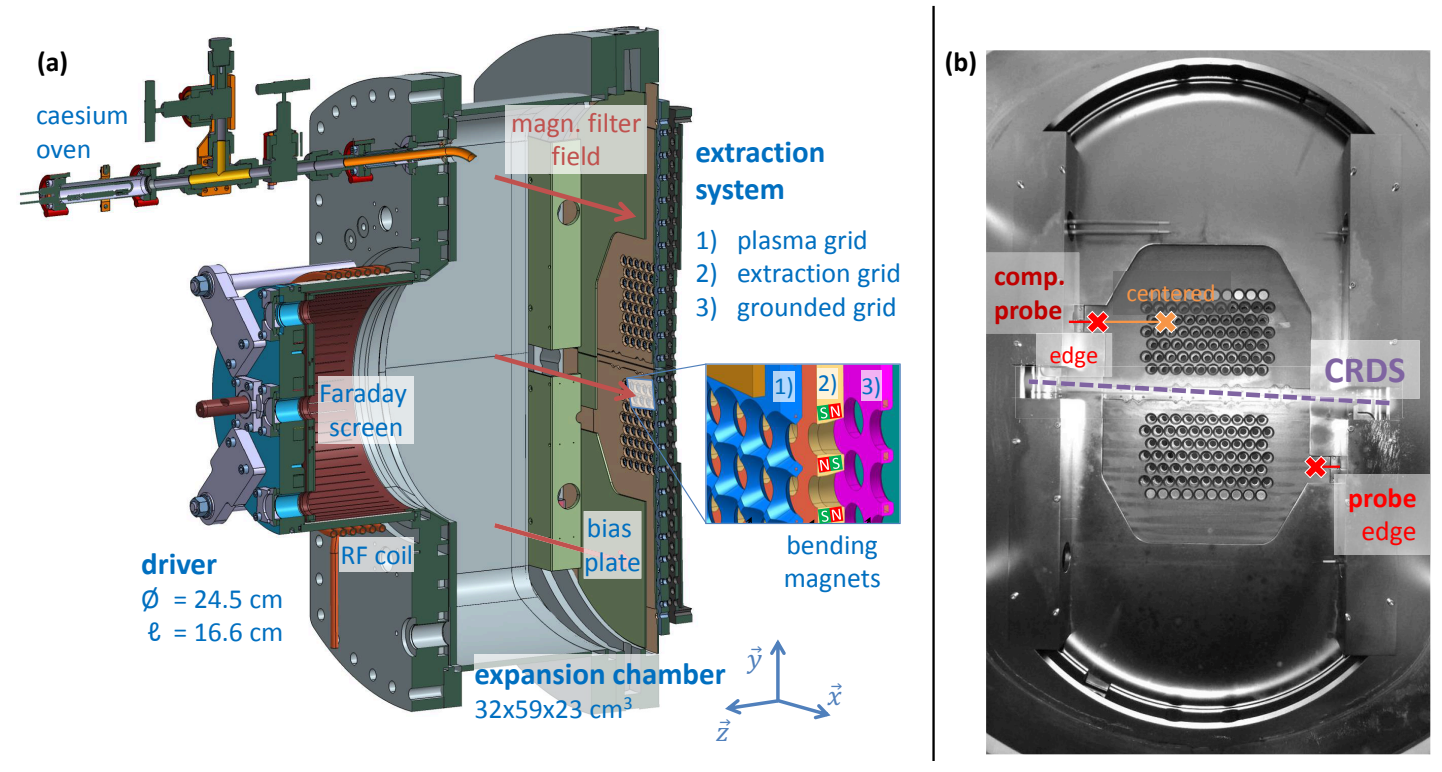

FIG. 1. (a): sketch of the ion source at BATMAN, (b): view onto the plasma grid showing the LOS of the CRDS and the measurement positions of the Langmuir probes.

A Cs oven is mounted in the top part of the source, which continuously evaporates Cs during the operational hours in order to optimize the source performance. The ceramic driver housing is shielded from the plasma by a Faraday shield. The line-of-sight (LOS) averaged $\mathrm{H}^{-}$density is measured by means of Cavity Ring-Down Spectroscopy (CRDS) ${ }^{21}$ at a vertically ( $\vec{y}$ direction) centered, horizontal ( $\vec{x}$ direction) LOS with a distance of $2.2 \mathrm{~cm}$ to the PG ( $\vec{z}$ direction). Two Langmuir probes are used, both with a distance of $0.7 \mathrm{~cm}$ to the PG: a RF compensated, moveable probe ${ }^{22}$ with $5 \mathrm{~mm}$ probe tip length and $100 \mu \mathrm{m}$ diameter is mounted in a horizontal port in the top part of the source. The probe is configured to measure at two positions each shot during the beam extraction phase: the inner position (centered) is in front of the extraction apertures and is used to determine the positive ion density as well as the plasma potential in front of the extraction area. The outer position (edge) is mainly used for comparison of the positive ion density with a vertically symmetric mounted, non RF compensated Langmuir probe at the bottom part (fixed position, $10 \mathrm{~mm}$ probe tip length, $300 \mu \mathrm{m}$ diameter). At a distance of $0.7 \mathrm{~cm}$ to the PG the measurement position of the Langmuir probes is still fairly deep in the bulk plasma: the plasma parameters ${ }^{22}$ of $T_{\mathrm{e}}=1 \mathrm{eV}, n_{\mathrm{e}}=1 \times 10^{16}-1 \times 10^{17} \mathrm{~m}^{-3}$ result in a Debye length of several $10 \mu \mathrm{m}$. The position very close to the PG is required for comparison of plasma 
parameters with extracted currents because theoretical models indicate that the complex plasma drift mechanisms influence plasma parameters and distribution close to the PG. ${ }^{15}$ The vertical distance $\Delta \vec{y}$ between both probes is $14 \mathrm{~cm}$, i.e. each probe is positioned in $7 \mathrm{~cm}$ distance from the vertical center.

For determining the positive ion density, the modified OML formula by $\mathrm{Chen}^{23}$ is used for fitting the recorded positive ion branch of the probe characteristics. The zero crossing of $\mathrm{d}^{2} I / \mathrm{d} U^{2}$ is used for the determination of the plasma potential. However, it is known that the determination of $\Phi_{\mathrm{pl}}$ with a single probe can be erroneous. ${ }^{24}$ In order to estimate the error bar of the determined plasma potential $\Phi_{\mathrm{pl}}$, the voltage difference between the maximum and the minimum of $\mathrm{d}^{2} I / \mathrm{d} U^{2}$ around the zero crossing is used, which is $2 \mathrm{~V}$ for all measurements. Thus, the error bar is $\Delta \Phi_{\mathrm{pl}}= \pm 1 \mathrm{~V}$. For the comparison of probe measurements with the source performance it should be kept in mind that the parameters determined with the Langmuir probe are measured locally whereas the electrically measured extracted current densities are globally averaged over the full area of the PG (126 apertures with a diameter of $0.8 \mathrm{~cm}$ yield an extraction area of $63.3 \mathrm{~cm}^{2}$ ). However, relative trends during parameter scans are still comparable.

The operational parameters have been $60 \mathrm{~kW}$ RF power, a pressure of $0.6 \mathrm{~Pa}$ and an extraction voltage of $5 \mathrm{kV}$ for all pulses shown in this paper. The $\mathrm{H}^{-}$source for ITER is required to operate at a pressure of $p \leq 0.3 \mathrm{~Pa}$ for minimizing stripping losses in the accelerator. ${ }^{1}$ However, in contrast to the standard magnetic filter field the setup with the external magnets does not allow stable plasma operation at $0.3 \mathrm{~Pa}$ at BATMAN. A moderate extraction voltage of $5 \mathrm{kV}$ has been chosen (for reaching high performance up to $10 \mathrm{kV}$ can be used) in order to reduce the heat load on the extraction grid and thus allowing a broader tolerable range for $j_{\mathrm{e}}$. The applied potentials to the different source components are listed in table I. A total high voltage of $-15 \mathrm{kV}$ is applied to the source body. The plasma grid is positively biased in the order of $20 \mathrm{~V}$ with respect to the source body. Since in this paper only the potentials in the plasma chamber are investigated, all shown potentials are shifted by $+15 \mathrm{kV}$ in the following for simplification, yielding to a potential of the source body of $0 \mathrm{~V}$ and of the plasma grid of $\approx+20 \mathrm{~V}$. In contrary to the standard operational scenario of BATMAN, in which a constant bias current is applied to the PG (making the source performance less dependent on operational parameters as the source pressure or the RF power $)^{6}$, the bias power supply has been configured to apply a constant bias voltage 
TABLE I. Potentials of the different source components during beam extraction.

\begin{tabular}{lc}
\hline \hline Source component & Potential \\
\hline Source body: driver, expansion chamber \& bias plate & $-15 \mathrm{kV}$ \\
Plasma grid & $\approx-14.98 \mathrm{kV}$ \\
Extraction grid & $-10 \mathrm{kV}$ \\
Grounded grid & $0 \mathrm{~V}$ \\
\hline \hline
\end{tabular}

during the full pulse length for all pulses shown in this paper.

The source has been conditioned with Cs for optimum performance for all measurements presented in this paper. In this case, the negative hydrogen ion density at an axial distance of some cm to the PG is similar to the electron density or even exceeds it. ${ }^{25,26}$

\section{BIAS BEHAVIOR}

The applied bias influences the plasma potential close to the PG due to the large size of the PG and the resulting large currents. The plasma potential $\Phi_{\mathrm{pl}}$ at the three probe positions as a function of the applied bias voltage $U_{\text {bias }}$ is plotted in figure 2 (a). For characterizing fluxes of charged particles towards and from the PG, the PG sheath potential difference $\Delta \Phi=U_{\text {bias }}-\Phi_{\mathrm{pl}}$ is of particular interest, which is shown in figure 2 (b). Here, the plasma potential measured by the upper probe at the centered position is used, which is the relevant position for comparison with extracted particle current densities.

The plasma potential is steadily increasing for higher applied bias voltages and a transition of the PG sheath property takes place in the applied bias voltage range: a low applied bias voltage leads to $U_{\text {bias }}-\Phi_{\mathrm{pl}}<0 \mathrm{~V}$ and thus electrons are (partly) repelled in the PG sheath from impinging the PG. In case of a high applied bias voltage, the PG sheath potential difference becomes $U_{\text {bias }}-\Phi_{\mathrm{pl}}>0 \mathrm{~V}$ and electrons are attracted towards the PG, whereas a potential barrier for $\mathrm{H}^{-}$produced on the surface of the $\mathrm{PG}$ arises. The PG sheath potential difference is about $\Delta \Phi=4.5 \mathrm{~V}$ at the highest applied bias. Since $\mathrm{H}$ atoms are the dominant conversion particle towards negative ions ${ }^{19}$ and their temperature is around $0.8 \mathrm{eV}^{27}$, most fraction of the converted $\mathrm{H}^{-}$can not penetrate this potential barrier. However, it must be taken into account that the plasma potential measurement is taken at one 


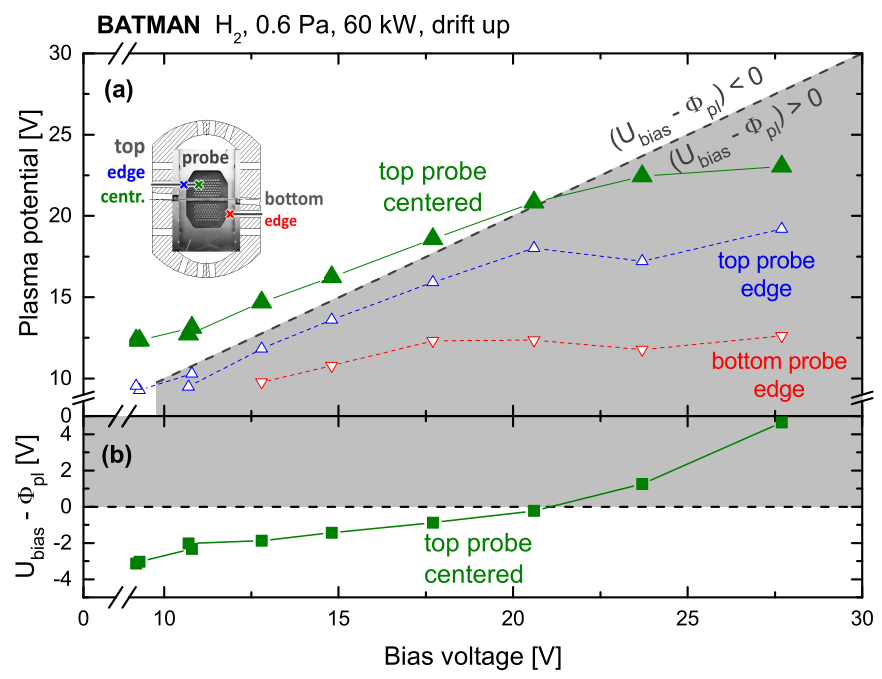

FIG. 2. Plasma potential at the three probe positions (a) and PG sheath potential difference (b) as a function of the applied bias voltage.

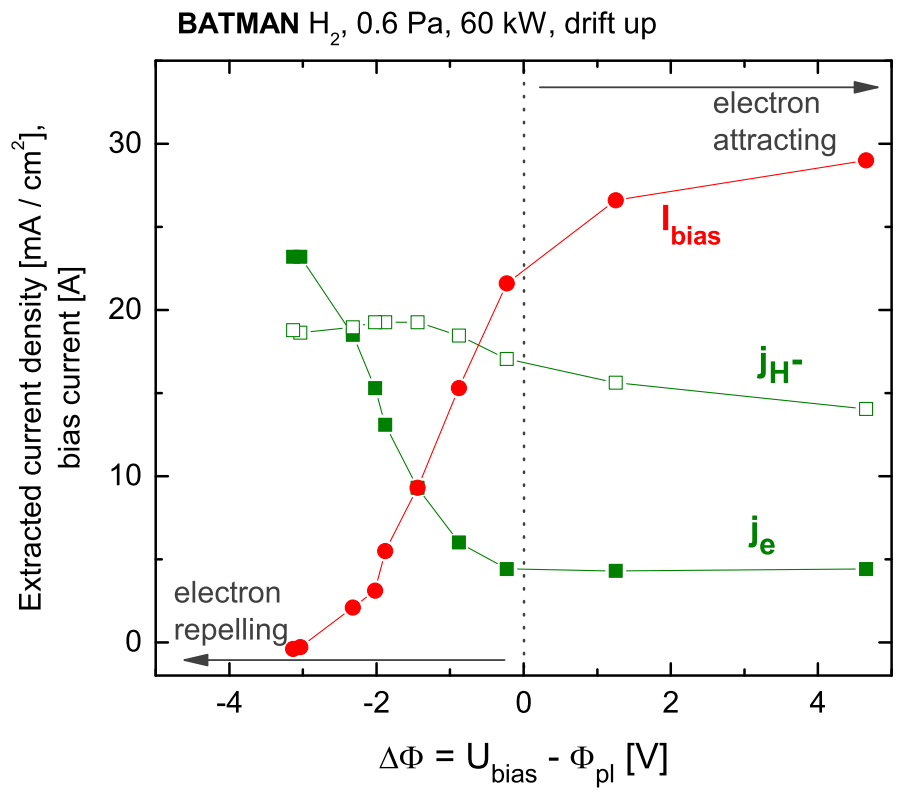

FIG. 3. Bias current $I_{\text {bias }}$, extracted $\mathrm{H}^{-}$current density $j_{\mathrm{H}^{-}}$and co-extracted electron current density $j_{\mathrm{e}}$ as a function of the PG sheath potential difference $\Delta \Phi=U_{\text {bias }}-\Phi_{\mathrm{pl}}$.

local position in front of the PG. As shown in figure figure 2 (a), the plasma potential varies in horizontal $(\vec{x})$ and vertical $(\vec{y})$ direction, thus the potential barrier for surface produced $\mathrm{H}^{-}$differs locally over the $\mathrm{PG}$ area.

The dependency of the resulting bias current $I_{\mathrm{bias}}, j_{\mathrm{e}}$ and $j_{\mathrm{H}^{-}}$on the PG sheath potential difference $\Delta \Phi$ is shown in figure 3. For increasing values of $\Delta \Phi$, the bias current is strongly 
increasing in the electron repelling sheath case, whereas it saturates in the electron attracting sheath case at a value of about $30 \mathrm{~A}$. The opposite behavior takes place for $j_{\mathrm{e}}$ : a strong reduction in the electron repelling case starting at $23 \mathrm{~mA} / \mathrm{cm}^{2}$ (corresponding to a total current of $1.5 \mathrm{~A}$ ) and a saturation in the electron attracting sheath case at a value of $4.4 \mathrm{~mA} / \mathrm{cm}^{2}$. Thus, $j_{\mathrm{e}}$ is decreased five times from the floating PG grid case (0 A bias current) towards the electron attracting sheath case. The co-extracted electron current is large when less electrons are removed through the sheath onto the PG. The extracted $\mathrm{H}^{-}$current density is almost stable for $\Delta \Phi<1 \mathrm{~V}$ at a value of $19 \mathrm{~mA} / \mathrm{cm}^{2}$ and starts decreasing for $\Delta \Phi>-1 \mathrm{~V}$ down to $14 \mathrm{~mA} / \mathrm{cm}^{2}$. This decrease of $j_{\mathrm{H}^{-}}$indicates that a significant fraction of extracted $\mathrm{H}^{-}$particles is produced on the $\mathrm{PG}$ between the extraction apertures (on the area which is not affected by the extraction meniscus formed due to the applied high voltage in front of each aperture). The transport of $\mathrm{H}^{-}$from this $\mathrm{PG}$ surface into the plasma depends on the PG sheath potential profile $\Delta \Phi$ and is hindered in case of the arising potential barrier. Taking into account $j_{\mathrm{e}}$ as well as $j_{\mathrm{H}^{-}}$, the optimum point of operation in this setup is an applied bias leading to a PG sheath potential difference of zero or slightly below $\left(U_{\text {bias }} \lesssim \Phi_{\text {pl }}\right)$; the bias current is then in the order of 20 A. Since the magnetic filter field acts as an electron filter, the optimum point of operation depends strongly on the magnetic filter field structure: with the standard filter field created by internal magnets, a much lower bias current of $1-2 \mathrm{~A}$ is required to lower $j_{\mathrm{e}}$ to its minimum and thus reach optimum performance, ${ }^{28}$ hence the optimum bias voltage is just slightly above the floating potential $\left(U_{\text {bias }} \gtrsim \Phi_{\text {fl }}\right)$ in this case.

The change of the positive ion density as a function of $\Delta \Phi$ is plotted in figure 4 (a). In general, the plasma density is a factor of 2.5-3 higher at the top centered position compared to the top edge position, almost independent of $\Delta \Phi$. However, the comparison between the top centered and the top edge position is not straight forward, since the edge position is located very close to the bias plate (see figure $1(\mathrm{~b})$ ). The positive ion density at the top edge position shows a small reduction for increasing values of $\Delta \Phi$ from $1.0 \times 10^{17} \mathrm{~m}^{-3}$ down to $7.3 \times 10^{16} \mathrm{~m}^{-3}$. At the bottom edge position $n_{\mathrm{i}+}$ is decreased even more from $9.5 \times 10^{16} \mathrm{~m}^{-3}$ to $5.0 \times 10^{16} \mathrm{~m}^{-3}$. This means that the plasma is distributed vertically more asymmetric with increasing value of the PG sheath potential difference. The change of the plasma symmetry is explained by the resulting variation of the axial ( $\vec{z}$ direction) electric field $\vec{E}$ close to the PG and thus change of the vertical $\vec{E} \times \vec{B}$ drift of the plasma. It should 


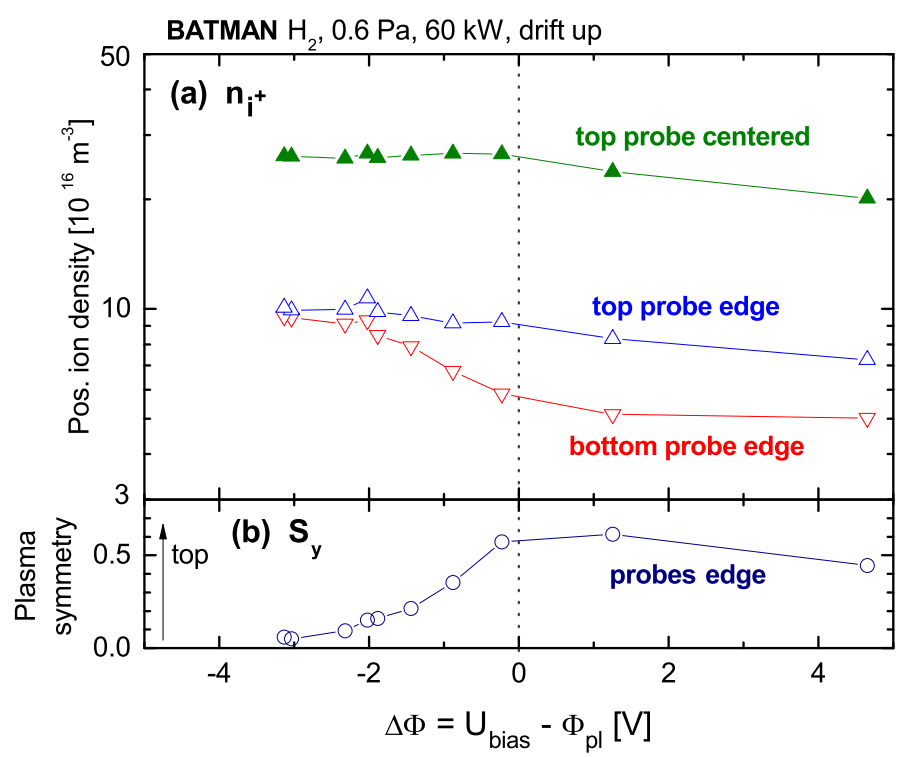

FIG. 4. (a): positive ion densities for varying PG sheath potential difference $\Delta \Phi$ at the three probe positions. (b): vertical plasma symmetry for varying $\Delta \Phi$, taken from measurements at the edge position.

be noted that during bias variation not only the local field in the PG sheath $\Delta \Phi$ is modified, but due to the strong influence of the bias on the plasma potential close to the PG (which will be shown in figure 5) also the global axial electric field in the expansion chamber towards the PG is changed. Thus, the change of the plasma asymmetry close to the PG is not only an effect of the varying PG sheath potential profile $\Delta \Phi$.

For quantification of the vertical plasma symmetry, the symmetry factor $S_{y}=\left(n_{\text {top }}-n_{\text {bot }}\right) /$ $\min \left(n_{\text {top }}, n_{\text {bot }}\right)$ is defined, in which the positive ion density of the top and bottom probe at the horizontal edge positions are used for calculation. In this definition a value of $S_{y}=0$ reflects perfect vertical symmetry whereas $S_{y}>0$ refers to a shift to the top part. The evolution of an almost symmetric vertical plasma symmetry at low values of $\Delta \Phi$ towards a stronger shift to the top (which is the drift direction) at higher values of $\Delta \Phi$ is shown in figure 4 (b). A strengthened plasma drift close to the plasma grid with increasing bias is regularly seen at BATMAN with the filter field created by external magnets. 


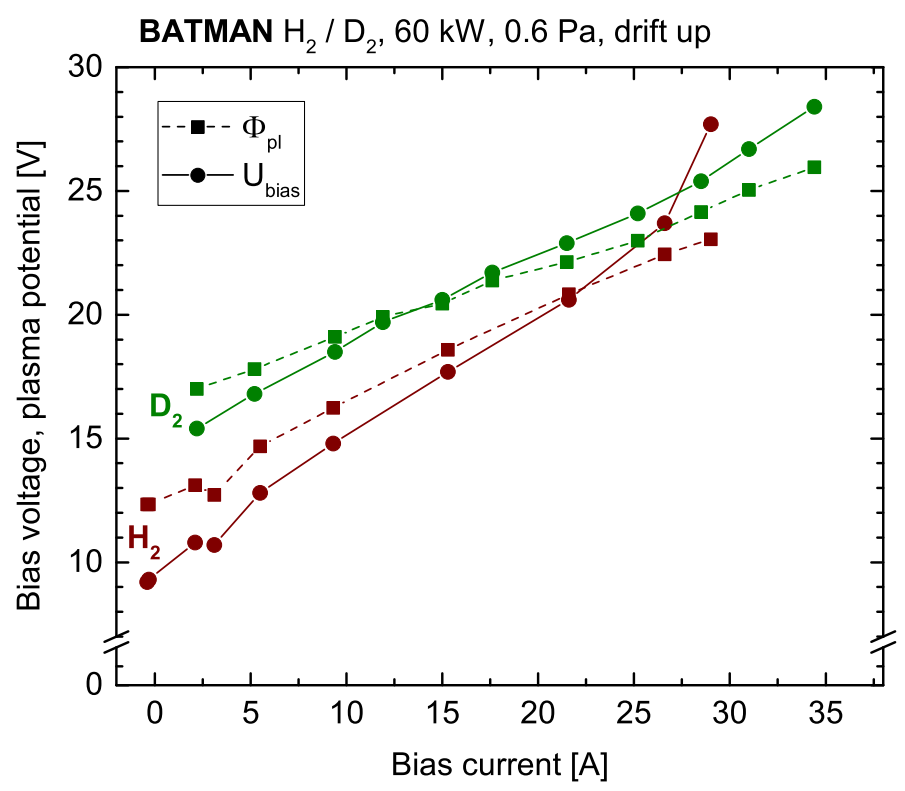

FIG. 5. Plasma potential and required bias voltage as function of bias current in hydrogen and deuterium operation.

\section{COMPARISON OF HYDROGEN AND DEUTERIUM OPERATION}

For the comparison of both isotopes, bias scans with a similar range of bias current $\left(I_{\text {bias }}=0-34 \mathrm{~A}\right)$ are compared. Since the source has been conditioned with caesium for optimum performance in both cases, a similar work function of the plasma grid is expected. The bias voltage, which is required to draw the bias current, as well as the plasma potential (top centered position) as a function of the bias current are shown in figure 5. Generally, the plasma potential as well as the bias voltage is larger in deuterium operation if comparing at the same bias current. This behavior is seen regularly at BATMAN. ${ }^{29}$ The comparison of $U_{\text {bias }}$ and $\Phi_{\mathrm{pl}}$ shows an intersection point for each isotope. This means that a transition in the PG sheath profile takes place in both isotopes in the applied bias range (electron repelling at low bias and electron attracting at high bias). The transition point is at a value of $U_{\text {bias }} \approx 21 \mathrm{~V}, I_{\text {bias }} \approx 22 \mathrm{~A}$ for hydrogen and $U_{\text {bias }} \approx 20 \mathrm{~V}, I_{\text {bias }} \approx 14$ A for deuterium - obviously, although the required bias current differs for both isotopes, the required bias voltage for the transition in the PG sheath is similar. The dynamics of charged particles in front of the PG depends strongly on the PG sheath potential difference. In order to investigate only the influence of the isotope, the comparison of both isotopes is always done as function of $\Delta \Phi$ in the following. 


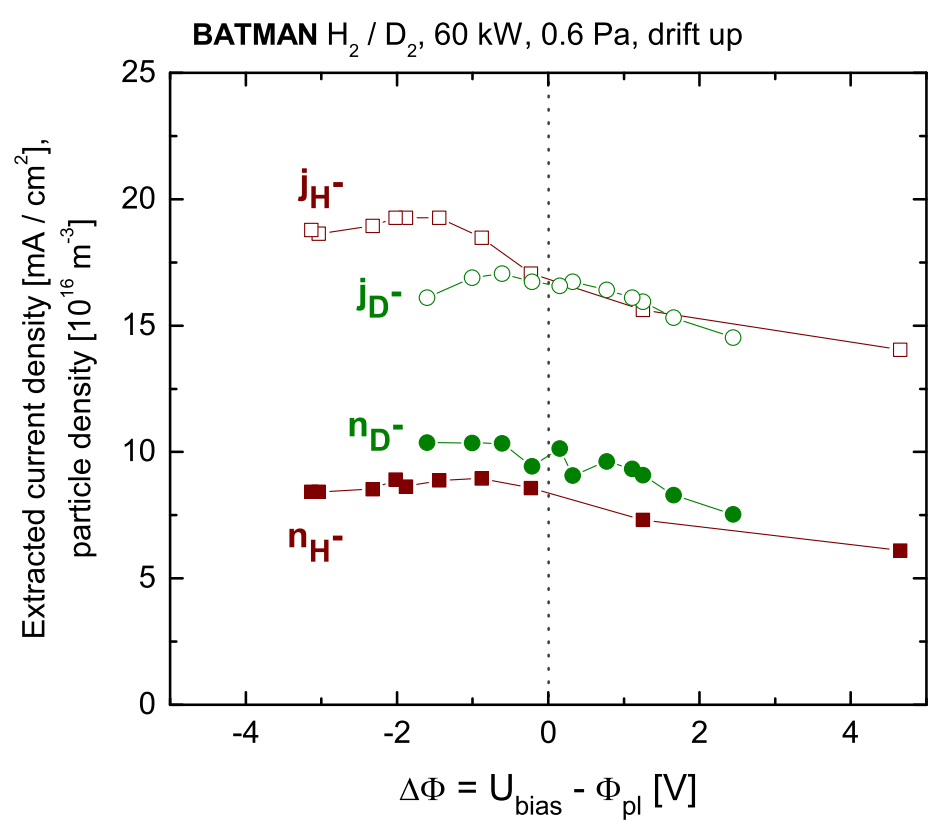

FIG. 6. Extracted negative ion current density and negative ion density as function of the plasma grid sheath potential difference in hydrogen and deuterium operation.

\section{A. Negative ion dynamics}

Before comparing the electron dynamics, the influence on negative ions is discussed. Figure 6 shows the extracted negative ion current density as well as the negative ion density in the plasma volume as a function of the PG sheath potential difference $\Delta \Phi$ for hydrogen and deuterium operation. The extracted $\mathrm{H}^{-}$current density $j_{\mathrm{H}^{-}}$is almost similar to $j_{\mathrm{D}^{-}}$, with the exception that at low values of $\Delta \Phi$ a larger extracted negative ion current density is reached in hydrogen operation. The negative ion density is higher in deuterium operation with a difference of roughly $2 \times 10^{16} \mathrm{~m}^{-3}$, independent on the value of $\Delta \Phi$. The dependence of $n_{\mathrm{H}^{-}}$and $n_{\mathrm{D}^{-}}$on $\Delta \Phi$ is similar to the one of $j_{\mathrm{H}^{-}}$and $j_{\mathrm{D}^{-}}$, which has been already discussed before.

The difference of the negative ion density between both isotopes is in good agreement with the characterization of the driver plasma, where an increased ratio of hydrogen atoms over molecules is found in deuterium operation compared to hydrogen operation: $n_{\mathrm{D}} / n_{\mathrm{D}_{2}}=0.49$ and $n_{\mathrm{H}} / n_{\mathrm{H}_{2}}=0.37 .{ }^{29}$ This increased ratio can at least partly compensate the doubled mass of deuterium and thus leads to a similar flux of deuterium and hydrogen atoms onto the plasma grid. As already mentioned before, atoms are the dominant conversion particle 
towards $\mathrm{H}^{-}$on the plasma grid; thus, a similar flux of surface produced negative ions is expected - if the positive ion density in front of the PG is sufficiently high for the required space charge compensation of $\mathrm{H}^{-}$. A similar flux of negative ions results then in an increased negative ion density in front of the PG in deuterium operation, again due to the doubled mass.

\section{B. Electron dynamics}

For the comparison of the electron dynamics between both isotopes, figure 7 (a) shows $j_{\mathrm{e}}$, (b) the resulting bias current, (c) the positive ion density at the top centered probe position and (d) the vertical plasma symmetry as a function of $\Delta \Phi$ for hydrogen and deuterium operation. The relative behavior of $j_{\mathrm{e}}$ is similar in hydrogen and deuterium operation and follows, as explained before, the transition from the electron repelling towards the electron attracting $\mathrm{PG}$ sheath. However, the absolute value of $j_{\mathrm{e}}$ is much higher in deuterium operation. In particular, the minimum value of $j_{\mathrm{e}}$ at optimum bias $(\Delta \Phi \approx 0 \mathrm{~V})$ is approximately a factor of seven higher in deuterium operation. The bias current shows a very similar behavior for both isotopes; however, the maximum bias current that can be drawn seems to be slightly higher in deuterium. This trend is in agreement with the increased positive ion density in deuterium operation $\left(\approx 5 \times 10^{17} \mathrm{~m}^{-3}\right)$ compared to hydrogen $\left(\approx 3 \times 10^{17} \mathrm{~m}^{-3}\right)$; for both isotopes there is no strong dependency on $\Delta \Phi$. The vertical plasma symmetry is similar in case of $\Delta \Phi<1 \mathrm{~V}$ for $\mathrm{H}_{2}$ and $\mathrm{D}_{2}$. As already mentioned before, the plasma asymmetry is not only affected by the local electrical field in the PG sheath connected to $\Delta \Phi$, but also by the axial electric field from the driver to the PG. The latter is different between both isotopes ${ }^{29}$, explaining differences in the vertical plasma symmetry.

In the following, the case with similar vertical plasma symmetry $(\Delta \Phi<1 \mathrm{~V})$ is discussed. The larger difference of the positive ion density between both isotopes (in the order of $10^{17} \mathrm{~m}^{-3}$ ) compared to the difference of the negative ion density (in the order of $10^{16} \mathrm{~m}^{-3}$ ) results due to quasi-neutrality in a larger electron density in deuterium operation. The latter leads to almost no change of the drawn bias current; however, $j_{\mathrm{e}}$ is strongly increased in deuterium in both sheath cases (electron repelling and electron attracting). The transport of magnetized electrons from the positions in the source which are accessible by diagnostics (several mm to the PG) to the plasma grid and thus counting to the bias current or the 


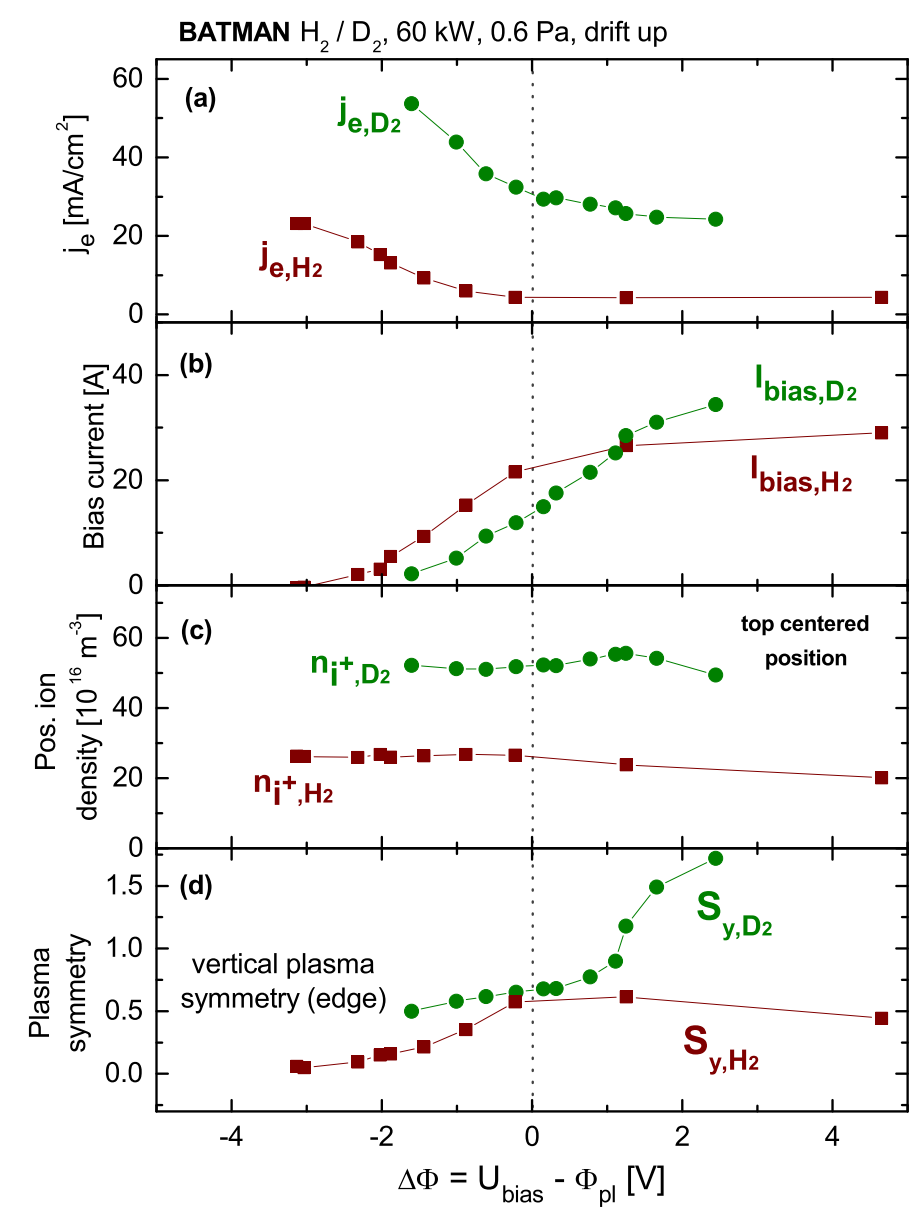

FIG. 7. Influence of the PG sheath potential difference on (a) the co-extracted electron current density, (b) the drawn bias current, (c) the positive ion density at the top centered position and (d) the vertical plasma symmetry for hydrogen and deuterium operation.

transport to the extraction meniscus and thus counting to $j_{\mathrm{e}}$ is complex due to the complex 3D structure of the total magnetic field, consisting of the filter field and the electron deflection field. ${ }^{30}$ Obviously, there must be a difference in this axial electron transport taking place between both isotopes, which is however not accessible for diagnostics. Gaining insight into the observed isotope difference may be a task for future modeling. Nevertheless, the relative behavior of the electron dynamics for varying bias is well described in the picture of reversing PG sheath potential difference for both isotopes.

It should be noted that at the required pressure of $0.3 \mathrm{~Pa}$ the co-extracted electron current density is higher compared to the case of 0.6 Pa presented in this paper. Although $j_{\mathrm{e}}$ can be lowered using the standard magnetic filter field configuration at the prototype 
source, applying an optimum bias is not sufficient for the reduction of $j_{\mathrm{e}}$ in order to reach the requirements for the ITER source in long deuterium pulses. ${ }^{7}$ Achieving a conditioning scenario in which the source can operate stable long pulses is still an important aim towards the negative hydrogen ion source for the ITER NBI.

\section{SUMMARY}

Lowering the amount of co-extracted electrons is of high importance in large-scale negative hydrogen ion sources for fusion since their amount can limit the source performance, in particular in deuterium operation. Biasing the plasma grid positively with respect to the source body is a well known knob for the reduction of $j_{\mathrm{e}}$. The influence of the bias on the plasma grid sheath potential difference $\Delta \Phi=U_{\text {bias }}-\Phi_{\mathrm{pl}}$ has been investigated; for this purpose the plasma potential in front of the PG has been determined by means of an RF compensated Langmuir probe. A clear transition from an electron repelling to an electron attracting sheath takes place for increasing bias. The co-extracted electron current reacts inverse to the resulting bias current: in the electron attracting sheath case, a large bias current is accompanied by a low co-extracted electron current and vice versa in the electron repelling sheath case. With the magnetic filter field created by external magnets, the optimum point of operation at BATMAN is to apply a bias which is close to the plasma potential in front of the PG or slightly below $\left(U_{\text {bias }} \lesssim \Phi_{\mathrm{pl}}\right)$, since for higher bias the extracted negative ion current is reduced.

A comparison between hydrogen and deuterium operation has been carried out regarding the negative ion and electron dynamics. Since $\Delta \Phi$ strongly influences the charged particle dynamics in front of the $\mathrm{PG}$, the comparison of both isotopes was done at the same value of $\Delta \Phi$ in order to investigate only the influence of the isotope. Due to the mass difference, a similar negative ion density is extracted in both isotopes since the negative ion density in the plasma volume is increased in deuterium operation by roughly $20 \%$. The plasma density in front of the PG is increased even more in deuterium by about $60 \%$, resulting in a similar value of the bias current but, however, in a strongly increased value of $j_{\mathrm{e}}$ (up to a factor of seven higher) in deuterium operation. As a consequence, biasing the PG is not sufficient in order to reduce $j_{\mathrm{e}}$ to a tolerable amount. 


\section{ACKNOWLEDGMENTS}

This work has been carried out within the framework of the EUROfusion Consortium and has received funding from the Euratom research and training program 2014-2018 under grant agreement No 633053. The views and opinions expressed herein do not necessarily reflect those of the European Commission.

\section{REFERENCES}

${ }^{1}$ R. Hemsworth, H. Decamps, J. Graceffa, B. Schunke, M. Tanaka, M. Dremel, A. Tanga, H. D. Esch, F. Geli, J. Milnes, T. Inoue, D. Marcuzzi, P. Sonato, and P. Zaccaria, Nuclear Fusion 49, 045006 (2009).

${ }^{2}$ B. Schunke, D. Bora, R. Hemsworth, and A. Tanga, AIP Conference Proceedings 1097, 480-490 (2009).

${ }^{3}$ A. Masiello, G. Agarici, T. Bonicelli, M. Simon, J. Alonso, M. Bigi, D. Boilson, G. Chitarin, C. Day, P. Franzen, S. Hanke, B. Heinemann, R. Hemsworth, A. Luchetta, D. Marcuzzi, J. Milnes, T. Minea, R. Pasqualotto, N. Pomaro, G. Serianni, W. Rigato, P. Sonato, V. Toigo, F. Villecroze, C. Waldon, and P. Zaccaria, Fusion Engineering and Design 86, 860-863 (2011).

${ }^{4}$ R. S. Hemsworth, A. Tanga, and V. Antoni, Review of Scientific Instruments 79, 02C109 (2008).

${ }^{5}$ M. Bacal and M. Wada, Applied Physics Reviews 2, 021305 (2015).

${ }^{6}$ E. Speth, H. Falter, P. Franzen, U. Fantz, M. Bandyopadhyay, S. Christ, A. Encheva, M. Fröschle, D. Holtum, B. Heinemann, W. Kraus, A. Lorenz, C. Martens, P. McNeely, S. Obermayer, R. Riedl, R. Süss, A. Tanga, R. Wilhelm, and D. Wünderlich, Nuclear Fusion 46, S220 (2006).

${ }^{7}$ W. Kraus, U. Fantz, P. Franzen, M. Fröschle, B. Heinemann, R. Riedl, and D. Wünderlich, Review of Scientific Instruments 83, $02 B 104$ (2012).

${ }^{8}$ U. Fantz, L. Schiesko, and D. Wünderlich, Plasma Sources Science and Technology 23, 044002 (2014).

${ }^{9}$ A. J. T. Holmes, Review of Scientific Instruments 53, 1517-1522 (1982). 
${ }^{10}$ K. N. Leung, K. W. Ehlers, and M. Bacal, Review of Scientific Instruments 54, 56-61 (1983).

${ }^{11}$ M. Bacal, J. Bruneteau, and P. Devynck, Review of Scientific Instruments 59, 2152-2157 (1988).

${ }^{12}$ F. E. Balghiti-Sube, F. G. Baksht, and M. Bacal, Review of Scientific Instruments 67, 2221-2227 (1996).

${ }^{13}$ Y. Takeiri, Y. Oka, M. Osakabe, K. Tsumori, O. Kaneko, T. Takanashi, E. Asano, T. Kawamoto, R. Akiyama, and T. Kuroda, Review of Scientific Instruments 68, 20032011 (1997).

${ }^{14}$ P. McNeely, M. Bandyopadhyay, P. Franzen, B. Heinemann, C. Hu, W. Kraus, R. Riedl, E. Speth, and R. Wilhelm, AIP Conference Proceedings 639, 90-111 (2002).

${ }^{15}$ S. Lishev, L. Schiesko, D. Wünderlich, and U. Fantz, AIP Conference Proceedings 1655, 040010 (2015).

${ }^{16}$ J. P. Boeuf, J. Claustre, B. Chaudhury, and G. Fubiani, Physics of Plasmas 19, 113510 (2012).

${ }^{17}$ C. Wimmer, U. Fantz, and NNBI-Team, AIP Conference Proceedings 1515, 246-254 (2013).

${ }^{18}$ R. McAdams, A. J. T. Holmes, D. B. King, and E. Surrey, Plasma Sources Science and Technology 20, 035023 (2011).

${ }^{19}$ D. Wünderlich, R. Gutser, and U. Fantz, Plasma Sources Science and Technology 18, 045031 (2009).

${ }^{20}$ P. Franzen, L. Schiesko, M. Fröschle, D. Wünderlich, U. Fantz, and the NNBI Team, Plasma Physics and Controlled Fusion 53, 115006 (2011).

${ }^{21}$ M. Berger, U. Fantz, S. Christ-Koch, and the NNBI Team, Plasma Sources Science and Technology 18, 025004 (2009).

${ }^{22}$ P. McNeely, S. V. Dudin, S. Christ-Koch, U. Fantz, and the NNBI Team, Plasma Sources Science and Technology 18, 014011 (2009).

${ }^{23}$ F. F. Chen, Plasma Sources Science and Technology 18, 035012 (2009).

${ }^{24}$ J. P. Sheehan, Y. Raitses, N. Hershkowitz, I. Kaganovich, and N. J. Fisch, Physics of Plasmas 18, 073501 (2011).

${ }^{25}$ S. Christ-Koch, U. Fantz, M. Berger, and the NNBI Team, Plasma Sources Science and Technology 18, 025003 (2009). 
${ }^{26}$ P. McNeely and L. Schiesko, Review of Scientific Instruments 81, $02 B 111$ (2010).

${ }^{27}$ D. Wünderlich, S. Mochalskyy, U. Fantz, P. Franzen, and the NNBI-Team, Plasma Sources Science and Technology 23, 015008 (2014).

${ }^{28}$ P. Franzen, H. Falter, U. Fantz, W. Kraus, M. Berger, S. Christ-Koch, M. Fröschle, R. Gutser, B. Heinemann, S. Hilbert, S. Leyer, C. Martens, P. McNeely, R. Riedl, E. Speth, and D. Wünderlich, Nuclear Fusion 47, 264 (2007).

${ }^{29}$ U. Fantz, L. Schiesko, D. Wünderlich, and the NNBI Team, AIP Conference Proceedings 1515, 187-196 (2013).

${ }^{30}$ R. Gutser, D. Wünderlich, U. Fantz, and the NNBI-Team, Plasma Physics and Controlled Fusion 51, 045005 (2009). 